\title{
Section introduction Introduction to sessions on 'Predicting personal risk for breast cancer'
}

Mitch Dowsett

Royal Marsden Hospital, Fulham Road, London SW3 6JJ, UK

Corresponding author: Mitch Dowsett, mitch.dowsett@icr.ac.uk

Published: 18 December 2008

This article is online at http://breast-cancer-research.com/content/10/S4/S9 (c) 2008 BioMed Central Ltd
Breast Cancer Research 2008, 10(Suppl 4):S9 (doi:10.1186/bcr2169)

normal, transforming and frankly malignant breast epithelial cells. There is unresolved controversy about whether direct carcinogenic effects of some oestrogen metabolites, which can clearly be demonstrated in model systems, also have a significant impact in women. Although this is largely unimportant in the potential use of plasma hormone levels for risk estimation, it would be a significant factor in selection of chemopreventive strategy, because oestrogen deprivation (for instance, aromatase inhibitors) would be expected to inhibit such an effect whereas oestrogen antagonists (for instance, tamoxifen) would not.

At present, despite its theoretical justification, measurement of plasma hormones has not been incorporated into risk algorithms. This is partly because of the paucity of well proven standardized assays for use in postmenopausal women and the need for application of such assays in very large sample sets. Such studies are needed to determine the interaction and independent significance of the hormone measurements alongside other more easily measured factors such as body mass index (BMI), which is correlated with plasma oestrogen levels in postmenopausal women as well as being a risk factor itself.

\section{Lifestyle}

There is persuasive epidemiological evidence that certain lifestyle factors, in addition to those associated with reproduction, are related to increased breast cancer risk. The majority of these factors relate to diet, with an emphasis on fat and overall calorific content. A clear characterization of risk determinants is not available because of the difficulty associated with quantifying dietary components and because the presence of one component in a diet is generally counterbalanced by the presence of another, making it impossible to ascribe risk confidently to an individual factor. $\mathrm{BMI}$, weight and weight gain may partly represent, and be useful as, an integrative measure of such dietary lifestyle, and they are much more amenable to measurement. nism underlying this is, at least in part, the mitogenic effect of the oestrogens on oestrogen receptor positive cells of 
The evidence that modifications to lifestyle have a significant effect in reducing breast cancer risk is also modest. Although the modifications such as reduced calories, and increased fruit and reduced fat consumption are those that would generally be considered to be helpful to general health, contemporary population behaviour has led to a worrying obesity epidemic, despite the evidence for this being a health risk. This suggests that there is a need for very clear evidence that risk reduction may result from lifestyle changes that have a significant effect on individual behaviour patterns.

\section{Genetics}

Over recent years a small number of highly penetrant genes, such as TP53 and BRCA1/BRCA2, have been discovered that profoundly increase the risk for breast cancer. The identification of strong pedigrees of breast cancer (with or without other cancer types) often leads to a search for causation by sequencing these genes. The demonstration of such a genetic aberration in a family and individuals therein can substantially alter personal risk to the extent that it makes consideration of other factors redundant. It is now widely accepted that it is highly unlikely that further genes of this type remain to be discovered. More recently, several moderate and many low penetrance genes have been identified (through genome-wide association studies); thus far, the indication is that incorporation of these only has slight effect on established risk profile algorithms. It is possible that the identification of more moderate/low penetrance genes may be discovered by research conducted within groups of breast cancers that are defined by phenotypic histopathological and/or molecular features (for example, oestrogen receptor positive or negative; lobular versus ductal).

\section{Integration of risk factors}

The possible integration of individual factors into risk algorithms is discussed in each of the sections above. Mammographic density is the most important factor that is not covered above, and this measure is readily available in the large majority of middle-aged women by virtue of their attendance at screening clinics. It may also be feasible to incorporate certain of the other factors, but to derive a tool that has sufficient certainty to have wide applicability will require comprehensive assessment of all potential factors in studies of many tens of thousands of women. Ideally, this work would be done within the UK National Health Service screening programme, such that it would be immediately transferable to that context and allow discussion of both breast cancer risk and possible interventions. This concept is widely recognized but progress in implementation is slow. In terms of the possible focusing of screening activity in those at greatest risk, it is disappointing to note that so far only about $5 \%$ of women can be identified as having less than $50 \%$ average risk.

\section{Psychosocial aspects}

The delivery of risk estimates to individuals is complicated by the degree of accuracy in public perception that risk is very variable. Of the strategies to communicate risk, diagrammatic representation has proven to be particularly helpful. It is of particular note that the framing of information can profoundly affect perception of risk. Although this fact may provide an opportunity to improve optimal clinical trial recruitment, it also imposes an ethical imperative upon the clinician to present data in such a way as to elicit the most accurate interpretation. A challenge for recruitment to trials of prevention remains achievement of greater acceptance by women at highest risk of intensive/invasive intervention, but also to address their lesser willingness to be randomized to markedly different strategies.

\section{Competing interests}

The author declares that they have no competing interests.

\section{Acknowledgements}

This article has been published as part of Breast Cancer Research Volume 10 Supplement 4, 2008: Controversies in Breast Cancer 2008. The full contents of the supplement are available online at http://breast-cancer-research.com/supplements/10/S4 